\title{
Cloning and molecular identification of triosephosphate isomerase gene from Apis cerana cerana and its role in response to various stresses
}

\author{
Yuli Zhou ${ }^{1}$, Fang $\mathrm{WANG}^{1}$, Feng $\mathrm{LiU}^{2}$, Chen $\mathrm{WANG}^{1}$, Yan $\mathrm{YAN}^{1}$, Xingqi Guo ${ }^{1}$, \\ Baohua $\mathrm{Xu}^{2}$
${ }^{1}$ State Key Laboratory of Crop Biology, College of Life Sciences, Shandong Agricultural University, Taian, Shandong 271018, People's Republic of China
${ }^{2}$ College of Animal Science and Technology, Shandong Agricultural University, Taian, Shandong 271018, People's Republic of China

Received 20 October 2015 - Revised 27 January 2016 - Accepted 12 February 2016

\begin{abstract}
Apis cerana cerana triosephosphate isomerase (AccTPI), a key regulatory enzyme in glycolysis and gluconeogenesis pathway, catalyzes the interconversion of glyceraldehyde 3-phosphate to dihydroxyacetone phosphate. However, its role in A. cerana cerana has not been completely clarified. In this study, a TPI gene was cloned from A. cerana cerana and named AccTPI. The open reading frame (ORF) of AccTPI is $744 \mathrm{bp}$, which encodes 247 amino acids with a predicted molecular weight and an isoelectric point of $26.8 \mathrm{kDa}$ and 8.42 , respectively. The expression of AccTPI was up-regulated by some abiotic stresses, while it was down-regulated by $\mathrm{HgCl}_{2}$, ultraviolet light (UV), and vitamin C (VC) treatment. Finally, a disc diffusion assay revealed that recombinant AccTPI proteins can protect cells from oxidative stresses. Taken together, all of the results indicate that AccTPI may play an important role in the metabolism, in antioxidant defense, and in many biological functions in the growth and development of A. cerana cerana .
\end{abstract}

\section{triosephosphate isomerase / Apis cerana cerana / abiotic stress / metabolism}

\section{INTRODUCTION}

As a pollinator, Apis cerana cerana is significant in keeping the balance between regional ecologies and agricultural economics (Weinstock et al. 2006). A. cerana cerana has been raised in Asian countries for thousands of years and has brought considerable economic benefits to the apicultural industry (Park et al. 2015). The honeybee is an important model organism for

Electronic supplementary material The online version of this article (doi:10.1007/s13592-016-0436-8) contains supplementary material, which is available to authorized users.

Corresponding author: X. Guo, xqguo@sdau.edu.cn; B.Xu, bhxu@sdau.edu.cn

Manuscript editor: Klaus Hartfelder neuroethological studies; it exhibits high behavioral plasticity and a remarkable ability to learn (Zhang et al. 2015). Compared with Apis mellifera, A. cerana cerana has actually been shown to learn better in a controlled laboratory setting (Qin et al. 2012). Recent colony losses of A. cerana cerana have thus raised concern, and possible explanations for bee decline include nutritional deficiencies and exposures to pesticides and pathogens. Thus, we chose $A$. cerana cerana as the experimental organism for this study. A. cerana cerana has unique characteristics including heat hardiness and disease resistance $(\mathrm{Li}$ et al. 2008). However, the survival of $A$. cerana cerana is under increasing threat due to various stressors including the indiscriminate use of pesticides and global warming. Moreover, organisms are exposed to a variety of unfavorable 
environmental stressors which are believed to induce the formation of reactive oxygen species (ROS) (Kottuparambila et al. 2012).

ROS, which include superoxide anions, hydrogen peroxide, and hydroxyl radicals, can be induced by external or internal factors, such as prooxidants, heavy metals, and pesticides (Imlay 2003). Generally, there is a balance between the generation of ROS and antioxidant processes, but exogenous stressors can break this balance (Brennan et al. 2008). High ROS concentrations contribute to degenerative diseases and aging through the oxidation of nucleic acids, proteins, and lipid membranes (Valko et al. 2006). To avoid the damage caused by ROS, insects have evolved an intricate mechanism of enzymatic antioxidant systems (Felton and Summers 1995). Reduced ROS generation, increased antioxidant protection or a combination of both increases the lifespan of social insects (Keller and Jemielity 2006). Aerobic organisms have also developed sophisticated antioxidant mechanisms and evolved various enzymatic antioxidant systems to avoid oxidative damage (Jia et al. 2014).

Metabolic regulation is very important for cellular tolerance to oxidants, providing the reducing power for the antioxidant machinery (Chu et al. 2008). Previous studies have demonstrated that triosephosphate isomerase (TPI) catalyzes the isomerization of glyceraldehyde-3-phosphate and dihydroxyacetone phosphate and plays an essential role in glycolysis, gluconeogenesis, and fatty acid synthesis (Zinsser et al. 2013). TPI plays an important function in generating energy and contributing to metabolism. Without TPI, there would be no net production of ATP to promote aerobic metabolism. Furthermore, the deficiency of TPI can result in metabolic diseases (Zhou et al. 2015).

Aerobic metabolism depends on the activities of glycosomal TPI and a mitochondrial glycerophosphate oxidase in Trypanosoma brucei (Helfert et al. 2001). During the life cycle of Clonorchis sinensis, adult worms can take in external glucose for their energy supply through the glycolysis pathway (Zhou et al. 2015). TPI deficiency in Drosophila melanogaster exhibits phenotypes such as susceptibility to infection, neurodegeneration, and reduced life span (Hrizo and Palladino 2010). The catalytic activity of TPI is unrestricted, and it is crucial to behavior and longevity (Roland et al. 2013). Yeast cells expressing a TPI variant are more resistant against oxidative stress and so TPI cannot tolerate all oxidative stress (Ralser et al. 2006). Furthermore, several studies demonstrate variations of TPI activity in relation to the levels of antioxidants in lymphocytes (Ralser et al. 2006). In bees, Wang et al. (2013) found that mixed-function oxidative enzymes are the most important enzymes in acaricide detoxification and resistance. Hence, we speculated that the TPI gene in bees might be associated with ROS, so we investigated the TPI antioxidant system in A. cerana cerana and its role in ROS defense mechanisms.

As the biochemical characterization of AccTPI was still unclear, this is the first study describing the cloning, overexpression, and characterization of a recombinant TPI from A. cerana cerana. The function of TPI in A. cerana cerana was studied to understand its role in cellular defense systems and metabolism. The RNA and protein expression levels of AccTPI were calculated. Furthermore, a disc diffusion assay was performed to evaluate the capacity of AccTPI to defend against oxidative stress.

\section{MATERIALS AND METHODS}

\subsection{Animals and various treatments}

A. cerana cerana were obtained from the experimental apiary at Shandong Agricultural University, Taian, China. Based on age, eye color, and shape, the worker honeybees were generally classified as larvae, pupae, and adults (Elias-Neto et al. 2010). The first (L1), third (L3), fifth (L5), and seventh (L7) day larvae; white-eyed (Pw), pink-eyed $(\mathrm{Pp})$, brown-eyed $(\mathrm{Pb})$, and dark-eyed $(\mathrm{Pd})$ pupae; and newly emerged adults (A1, 1 day post-emergence), 1 week-old bees (A7, usually 7-10 days postemergence), which are likely nurses, and 2 week-old bees (A15, older than 15 days post-emergence), which can be foragers, were gathered during the spring season from two hives maintained in the experimental apiary. For tissue-specific analyses of AccTPI expression levels, the antenna (AN), abdomen (AB), head (HE), hemolymph (HA), epidermis (EP), venom gland (VG), muscle (MU), thoracic (TH), and midgut (MI) of adult workers were used. These were dissected on ice, washed twice with PBS, frozen in liquid nitrogen, and stored at $-70^{\circ} \mathrm{C}$. 
Fifteen-day-old adult bees were collected randomly and reared under $34{ }^{\circ} \mathrm{C}$ and $60 \%$ relative humidity and fed a diet of water and a combination of pollen and sucrose (Alaux et al. 2010). The worker honeybees were randomly separated into eight groups, and each group contained 30 individuals. Groups 1, 2, and 3 were exposed to 4,14 , and $44{ }^{\circ} \mathrm{C}$, respectively. Group 4 was treated with ultraviolet light (UV) $(254 \mathrm{~nm}$, $30 \mathrm{~mJ} / \mathrm{cm}^{2}$ ). Groups 5 and 6 were exposed to two pesticides $(2.0 \mathrm{mg} / \mathrm{L}$ of paraquat and pyridaben was added to the basic adult diet, respectively). The control group was fed on a normal diet containing water, $70 \%$ powdered sugar, and $30 \%$ honey from the source colonies. Group 7 was fed $\mathrm{HgCl}_{2}(3 \mathrm{mg} / \mathrm{mL})$. Group 8 was subjected to vitamin C $(20,000 \mathrm{mg} / \mathrm{kg})$. The honeybees were flash-frozen in liquid nitrogen at a suitable time and stored at $-70{ }^{\circ} \mathrm{C}$ until use.

\subsection{RNA extraction, cDNA synthesis, and DNA preparation}

Total RNA was extracted from adult bees with the TRIzol reagent (Invitrogen, Carlsbad, CA, USA), and the RNA was treated with RNase-free DNase I (Promega, Madison, WI, USA). Then, the cDNA was synthesized using a reverse transcription system (TransGen Biotech, Beijing, China). The genomic DNA was isolated from the honeybees with the EasyPure Genomic DNA Extraction Kit (TransGen Biotech, Beijing, China).

\subsection{Primers and PCR procedure}

The primer sequences and PCR procedure are listed in Tables I and II, respectively.

\subsection{Bioinformatics analysis and phylogenetic tree construction}

The conserved nucleotide sequences of AccTPI were analyzed using DNAman software 6.0.3 and the National Center for Biotechnology Information (NCBI) bioinformatics tools (http://blast.ncbi.nlm.nih.gov/ Blast.cgi). The predicted amino acid sequence was aligned with the homologous sequences of various insects, which were acquired from the NCBI Genbank database. The molecular weight and theoretical isoelectric point were predicted using the online tool ExPASy (http://web.expasy.org/compute_pi/). A phylogenetic tree was constructed using the Molecular Evolutionary Genetic Analysis (MEGA version 4.0) software.

\subsection{Quantitative real-time PCR}

To evaluate the expression profile of AccTPI in different developmental stage tissues and abiotic stresses, RT-qPCR was performed using the SYBR ${ }^{\circledR}$ PrimeScript ${ }^{\text {TM }}$ RT-PCR Kit (TaKaRa, Dalian, China) with two specific primers (DLF and DLR). The reaction volume and the reaction requirements were as those of Yao et al. 2013. The PCR reactions were performed using a CFX96 ${ }^{\mathrm{TM}}$ Real-Time System (Bio-Rad), and the $\beta$-actin gene (GenBank accession no. HM640276; A. cerana cerana actin-related protein 1) was used as an internal control. We also obtained experimental data in which we tested and compared additional reference genes ( $\beta$-actin, ribosomal protein 49 , elongation factor 1-alpha, tbp-association factor); see Supplemental Fig. 3. All of the samples were analyzed in triplicate. The relative gene expression levels of AccTPI were calculated using the $2^{-\Delta \Delta \mathrm{Ct}}$ comparative CT method (Livak and Schmittgen 2001). The analysis was performed with Duncan's multiple range test using Statistical Analysis System (SAS) software version 9.1.

\subsection{Protein expression and purification of recombinant AccTPI}

To obtain the AccTPI protein, the recombinant plasmid pET-30a (+)-AccTPI was transfected into Escherichia coli BL21 (DE3). The bacteria were incubated in LuriaBertani (LB) broth with kanamycin at $37{ }^{\circ} \mathrm{C}$ until the cell density at $600 \mathrm{~nm}$ reached $0.4-0.6$. Then, the AccTPI expression was induced with $1.0 \mathrm{mM}$ isopropyl-1-thio- $\beta$ D-galactopyranoside (IPTG) at $18{ }^{\circ} \mathrm{C}$ overnight. The protein was purified on a HisTrap ${ }^{\mathrm{TM}} \mathrm{FF}$ column (GE Healthcare, Uppsala, Sweden). The expression of the target protein was analyzed by $12 \%$ sodium dodecyl sulfate polyacrylamide gel electrophoresis (SDS-PAGE).

\subsection{Western blotting analysis}

The white mice for the generation of antibodies came from China Biologic Products, Inc. (Shandong). The protocol numbers of certificates that they produced and used for animal research were SCXK (LU) 20130006 and SYXK (LU) 2013 0011, respectively. The Tissue 
Table I. The primers in this study.

\begin{tabular}{lll}
\hline Abbreviation & Primer sequence (5'-3') & Description \\
\hline TF & GGCCATGGGTCGTAAATTTTTTG & cDNA sequence primer, forward \\
TR & CATCTCACTGCTTAGCGTTAACG & cDNA sequence primer, reverse \\
5 TW & TGCAACCTTATATGTATTTTGTCAGC & 5'RACE reverse primer, outer \\
5 TN & TGCAACCTTATATGTATTTTGTCCAGC & 5'RACE reverse primer, inner \\
3 TW & GTAGCCCATGCTTTAGATAGTGGAC & 3'RACE forward primer, outer \\
3 TN & CACACCACAACAAGCTCAAGAG & 3'RACE forward primer, inner \\
AAP & GGCCACGCGTCGACTAGTAC(G) $)_{14}$ & Abridged anchor primer \\
AUAP & GGCCACGCGTCGACTAGTAC & Abridged universal amplification primer \\
B25 & GACTCTAGACGACATCGA & 3'RACE universal primer, outer \\
B26 & GACTCTAGACGACATCGA(T) ${ }_{18}$ & 3'RACE universal primer, inner \\
DLF & TGTTGAGGTTGTCGTTGGAGTAC & Real-time PCR primer, forward \\
DLR & GGACTTATTTCTCCAGTAAATGCTC & Real-time PCR primer, reverse \\
$\beta-\mathrm{s}$ & TTATATGCCAACACTGTCCTTT & Standard control primer, forward \\
$\beta-x$ & AGAATTGATCCACCAATCCA & Standard control primer, reverse \\
G1 & GGGGATTTCATAAAATCGGGTTGG & Genomic sequence primer, forward \\
G2 & GGTTATGTGATTACTTATGCACTATATTTGTG & Genomic sequence primer, reverse \\
YHF & GATATCATGGGTCGTAAATTTTTGTTGGTGG & Protein expression primer, forward \\
YHR & CGAGCTCTCACTGCTTAGCGTTAACGAT & Protein expression primer, reverse \\
\hline
\end{tabular}

Protein Extraction Kit (CoWin Bioscience Co., Beijing, China) was used to extract the total A. cerana cerana protein, and it was quantified with the BCA Protein Assay Kit (Nanjing Jiancheng Bioengineering Institute, Nanjing, China). Western blotting was performed according to the procedure of Chen et al. (2014). The following treatments were performed to analyze the protein expression levels: (1) $4{ }^{\circ} \mathrm{C}$, (2) $\mathrm{H}_{2} \mathrm{O}_{2}$, (3) methomyl, (4) pyridaben, (5) UV, and (6) vitamin C (VC).

\subsection{Disc diffusion assay}

Disc diffusion assays were performed according to Chen et al. (2014) for the evaluation of the activity of recombinant AccTPI. E. coli BL21 (DE3) cells were incubated in LB liquid medium with kanamycin at $37{ }^{\circ} \mathrm{C}$ until the cell density reached 0.4-0.6 $\mathrm{OD}_{600}$, then IPTG was introduced to the $\mathrm{LB}$ broth at a final concentration of $1 \mathrm{mM}$.

Table II. PCR amplification conditions.

\begin{tabular}{|c|c|}
\hline Primer pair & Amplification conditions \\
\hline TF/TR & 10 min at $94{ }^{\circ} \mathrm{C}, 40 \mathrm{~s}$ at $94{ }^{\circ} \mathrm{C}, 40 \mathrm{~s}$ at $54^{\circ} \mathrm{C}, 60 \mathrm{~s}$ at $72{ }^{\circ} \mathrm{C}$ for 35 cycles, 10 min at $72{ }^{\circ} \mathrm{C}$ \\
\hline 5TW/AAP & 10 min at $94{ }^{\circ} \mathrm{C}, 40 \mathrm{~s}$ at $94^{\circ} \mathrm{C}, 40 \mathrm{~s}$ at $54^{\circ} \mathrm{C}, 60 \mathrm{~s}$ at $72{ }^{\circ} \mathrm{C}$ for 35 cycles, 10 min at $72{ }^{\circ} \mathrm{C}$ \\
\hline 5TN/AUAP & 10 min at $94{ }^{\circ} \mathrm{C}, 40 \mathrm{~s}$ at $94{ }^{\circ} \mathrm{C}, 40 \mathrm{~s}$ at $55^{\circ} \mathrm{C}, 60 \mathrm{~s}$ at $72{ }^{\circ} \mathrm{C}$ for 35 cycles, 10 min at $72{ }^{\circ} \mathrm{C}$ \\
\hline 3TW/B26 & 10 min at $94{ }^{\circ} \mathrm{C}, 40 \mathrm{~s}$ at $94{ }^{\circ} \mathrm{C}, 40 \mathrm{~s}$ at $57^{\circ} \mathrm{C}, 60 \mathrm{~s}$ at $72{ }^{\circ} \mathrm{C}$ for 35 cycles, 10 min at $72{ }^{\circ} \mathrm{C}$ \\
\hline $3 \mathrm{TN} / \mathrm{B} 25$ & 10 min at $94{ }^{\circ} \mathrm{C}, 40 \mathrm{~s}$ at $94{ }^{\circ} \mathrm{C}, 40 \mathrm{~s}$ at $55^{\circ} \mathrm{C}, 60 \mathrm{~s}$ at $72{ }^{\circ} \mathrm{C}$ for 35 cycles, 10 min at $72{ }^{\circ} \mathrm{C}$ \\
\hline G1/G2 & 10 min at $94{ }^{\circ} \mathrm{C}, 40 \mathrm{~s}$ at $94{ }^{\circ} \mathrm{C}, 40 \mathrm{~s}$ at $55^{\circ} \mathrm{C}, 120 \mathrm{~s}$ at $72{ }^{\circ} \mathrm{C}$ for 35 cycles, 10 min at $72{ }^{\circ} \mathrm{C}$ \\
\hline YHF/YHR & 10 min at $94{ }^{\circ} \mathrm{C}, 40 \mathrm{~s}$ at $94^{\circ} \mathrm{C}, 40 \mathrm{~s}$ at $58^{\circ} \mathrm{C}, 60 \mathrm{~s}$ at $72^{\circ} \mathrm{C}$ for $35 \mathrm{cycles}, 10 \mathrm{~min}$ at $72{ }^{\circ} \mathrm{C}$ \\
\hline
\end{tabular}


They were induced using IPTG and incubated for $8 \mathrm{~h}$ at $18{ }^{\circ} \mathrm{C}$ with shaking at $180 \mathrm{rpm}$. Approximately $5 \times 10^{8}$ bacterial cells overexpressing AccTPI were seeded onto LB-kanamycin agar plates and incubated at $37{ }^{\circ} \mathrm{C}$ for $1 \mathrm{~h}$. Filter discs (8-mm diameter) soaked with different concentrations of $t$-butylhydroperoxide $(0,15,20,30$, or $40 \mathrm{mM})$ and cumene hydroperoxide $(0,30,50$, 80 , or $100 \mathrm{mM}$ ) were placed on the surface of the top agar. Eventually, the cells were incubated at $37{ }^{\circ} \mathrm{C}$ for $24 \mathrm{~h}$ before the inhibition zones around the paper discs were measured as described by Burmeister et al. (2008). E. coli BL21 cells transformed with empty pET-30a(+) were used as the control. The greater of the bacteriostatic circle bacteria is more sensitive, rather than vise versa.

\section{RESULTS}

\subsection{Identification of the full-length cDNA and sequence analysis}

The full-length cDNA of the AccTPI gene (GenBank accession no. KP994676) was cloned. It comprised 1894 bp containing a 744-bp open reading frame (ORF), a 158-bp 5'-untranslated region (UTR), and a 992-bp 3'-UTR. The ORF encodes a polypeptide of 247 amino acids with a predicted molecular weight of $26.8 \mathrm{kDa}$ and theoretical isoelectric point of 8.42 .

Multiple sequence alignments of several TPI proteins indicates that the putative AccTPI shares high homology with A. mellifera AmTPI (NP 001090623.1), Megachile rotundata MrTPI (XP 003700534.1), Harpegnathos saltator HsTPI (XP 011146748.1), and Acromyrmex echinatior AeTPI (EGI64718.1), respectively (Figure 1a). As shown in Figure 1, we found one TPI locus and several protein kinase $\mathrm{C}$ phosphorylation sites in the ORF. A phylogenetic tree was built to illustrate the evolutionary relationship of TPI among insects. The exons and introns of AccTPI were aligned with AmTPI (NM 001097154.2), MrTPI (XM 003700486.1), and HsTPI (XM 011148446.1). This analysis showed that AccTPI, AmTPI, MrTPI, and HsTPI contain two introns and three exons (Supplemental Fig. 1).

\subsection{Developmental and tissue-specific ex- pression patterns of AccTPI}

To investigate the expression patterns at different developmental stages and specific tissues, RT-qPCR assays were performed. As shown in Figure 2a, the expression levels peaked in the forager stage. The mRNA expression levels of AccTPI are higher in the L3 larval stages. Additionally, the expression levels in different AccTPI tissues were higher in the abdomen and thorax (Figure 2b).

\subsection{Expression patterns of AccTPI under abiotic stresses}

To verify whether abiotic stresses could affect AccTPI expression, a 15-day postemergence adult stage was chosen and total RNA was extracted. The expression of AccTPI was induced by $4{ }^{\circ} \mathrm{C}$ treatment and reached the highest level at $2 \mathrm{~h}$ after exposure. Moreover, the expression of AccTPI was inhibited by $14{ }^{\circ} \mathrm{C}$ treatment and the expression level subsequently increased after $44{ }^{\circ} \mathrm{C}$ treatment (Figure $3 \mathrm{a}-\mathrm{c}$ ). The expression of AccTPI was up-regulated in response to the paraquat and pyridaben treatments (Figure $3 \mathrm{~d}$, e). In contrast, the expression of AccTPI was down-regulated after $\mathrm{UV}, \mathrm{HgCl}_{2}$, and $\mathrm{VC}$ treatments (Figure $3 \mathrm{f}-\mathrm{h}$ ).

\subsection{Expression and purification of recombinant AccTPI protein}

To further characterize the properties of AccTPI via protein levels, AccTPI was transformed into $E$. coli BL21 (DE3) with the pET-30a(+) vector. The E. coli cells were obtained from the LB solution after induction with IPTG overnight. SDS-PAGE analysis indicated that a recombinant protein was expressed and had a molecular mass of approximately $33 \mathrm{kDa}$ (Supplemental Fig. 2, lane 2). It contained the AccTPI protein $(26.8 \mathrm{kDa})$ and the His tag $(7 \mathrm{kDa})$. The recombinant protein was purified with HisTrap $^{\text {TM }}$ FF columns. The result is shown in Supplemental Fig. 2, lane 7. 
a

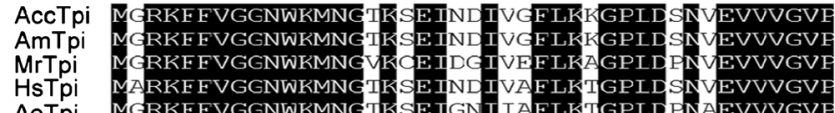

HsTpi

AeTpi

RKE FVGGNWKMNC

AccTpi

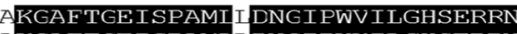

\section{$\bar{\Lambda} \mathbf{\Delta}$}

AccTpi VAETVRIIYGGSVTAGNARLLAKERDILGFLVGGASLKFDFVCIVNAK

AmTpi VAETYRIIYGGSVTAGNAKLLAKEKDILGE LVGGASLKEDEVCূIVNAK

MrTpi VAENVRIIYGGSVTAGNAKLLAKEKDILGFLVGGASLKEDFVCূIVNAK

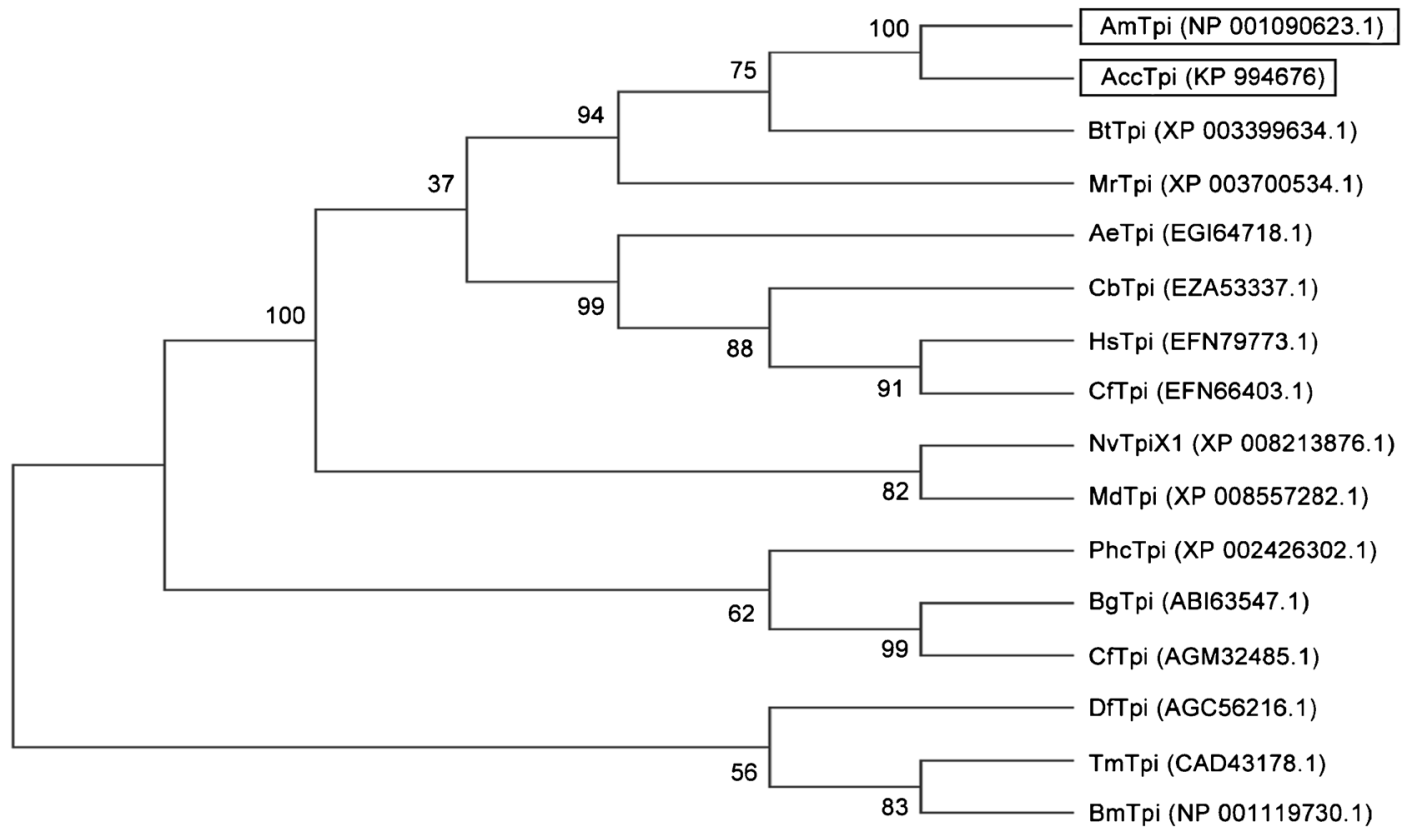

Figure 1. Multiple alignment analysis of AccTPI and a phylogenetic tree of the TPI amino acid sequences of several species. a Alignment of the deduced AccTPI protein sequence with other known species of TPI. The amino acid sequences of AmTPI, MrTPI, HsTPI, and AeTPI were used in the analysis. The TPI locus is marked by a horizontal line. The protein kinase $\mathrm{C}$ phosphorylation sites are marked by a black triangle. $\mathbf{b}$ Phylogenetic tree analysis of TPI from different species. The amino acid sequences of TPI were all obtained from NCBI.

\subsection{Western blot analysis}

A western blot analysis was performed to further detect the expression profiles of AccTPI under various adverse stresses. The results displayed that the expression of AccTPI protein levels were consistent with the transcriptional expression levels. The expression levels of AccTPI 

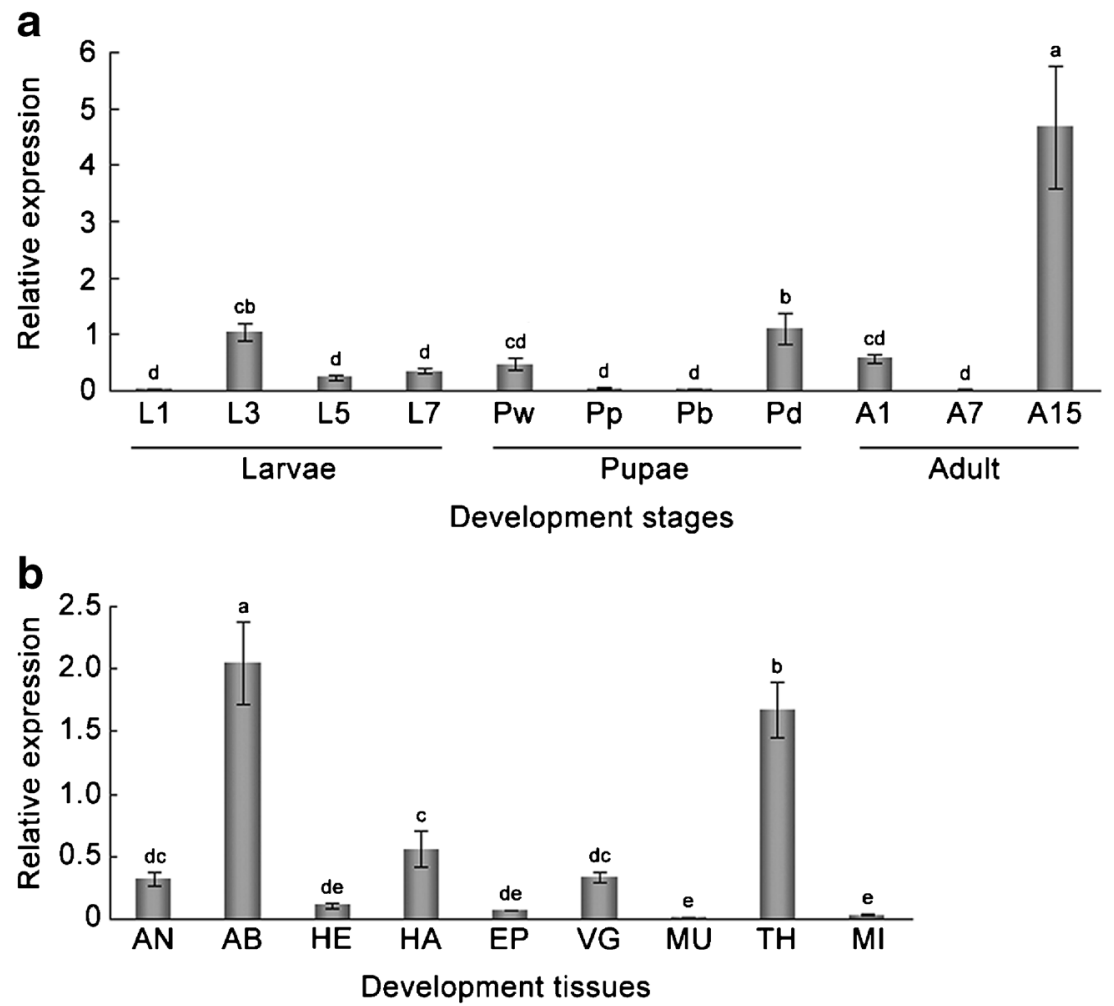

Figure 2. Expression profile of AccTPI in different developmental stages and tissues. a The expression profile of AccTPI was examined by RT-qPCR in different developmental stages including larvae, pupae, newly emerged adult workers, nurses, and foragers. b The expression profile of AccTPI was studied by RT-qPCR in various tissues. The data are the mean \pm SEM of three independent experiments. The letters above the columns indicate significant differences $(P<0.01)$ according to Duncan's multiple range test.

were up-regulated by $4{ }^{\circ} \mathrm{C}, \mathrm{H}_{2} \mathrm{O}_{2}$, and methomyl and pyridaben treatments. Furthermore, the expression levels of AccTPI were down-regulated by UV and VC treatments (Figure 4).

\subsection{Disc diffusion assay under various stresses}

To provide direct evidence that AccTPI is responsible for antioxidant defenses, a disc diffusion assay was performed according to Zhang et al. (2013). After overnight exposure to various stressors, the halo diameters of the death zones were significantly different for the various treatment concentrations. Compared with plates containing the control bacteria, the inhibition zones around the stressorsoaked filters were smaller in diameter containing E. coli overexpressing AccTPI (Figure 5).

\section{DISCUSSION}

Sequence alignment and the phylogenetic tree analysis showed that AccTPI has a high degree of homology with several representative TPI amino acid sequences (Figure 1). Taken together, these results indicate that the gene we isolated is a bona fide AccTPI.

To better understand its physiology, AccTPI expression at different development stages and in various tissues was analyzed. The expression of AccTPI reached a maximum in the 15-day post-emergence workers, which likely are foragers (Figure 2a). TPI, a key regulatory enzyme of glycolysis and gluconeogenesis, plays an 

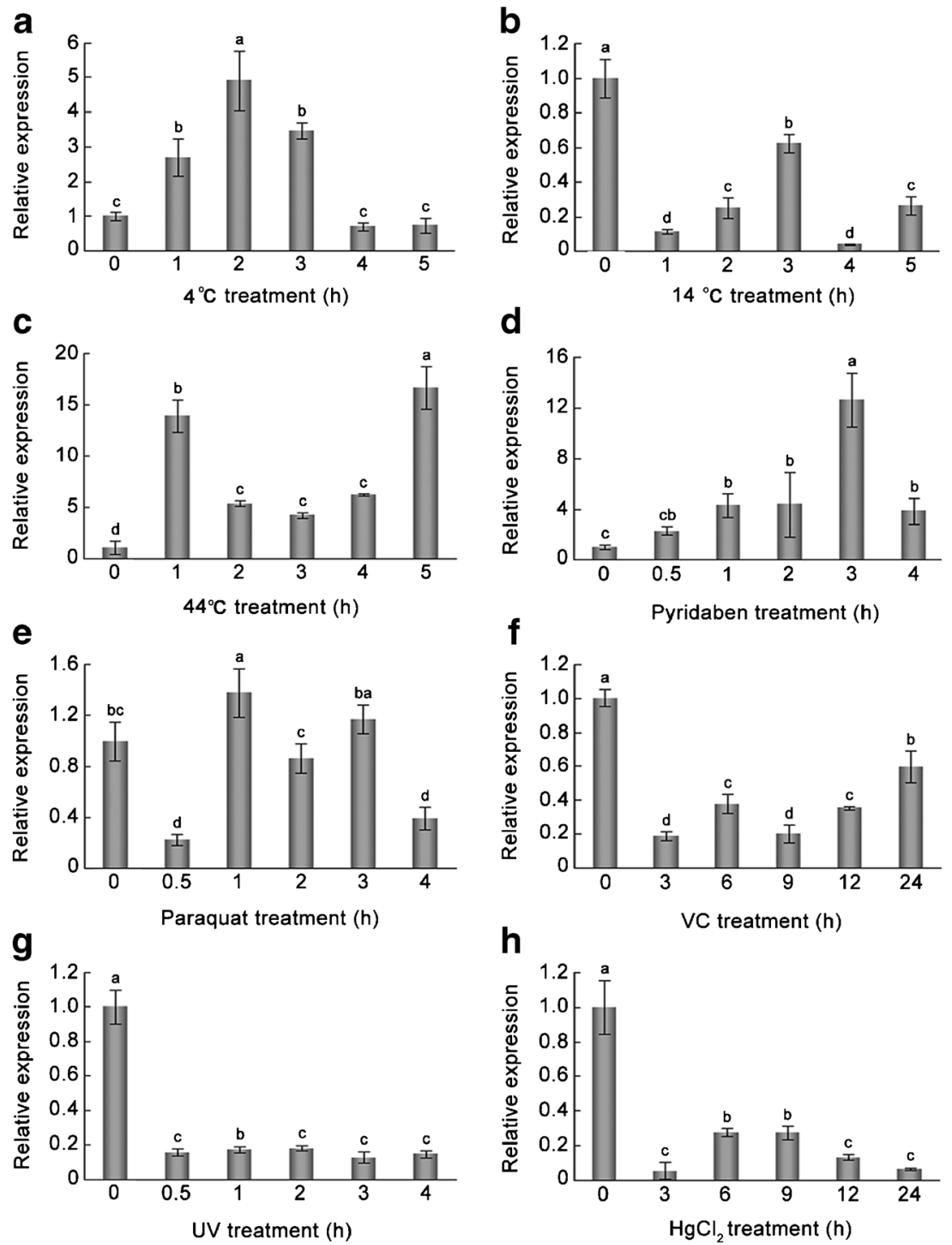

Figure 3. Expression profile of AccTPI under different abiotic stresses. The expression of AccTPI was analyzed by RT-qPCR using total RNA extracted from $A$. cerana cerana, which was treated with different stressors. For the stress treatments, 15 -day-old adult bees were subjected to treatments with a $4{ }^{\circ} \mathrm{C}, \mathbf{b} 14{ }^{\circ} \mathrm{C}, \mathbf{c ~} 44^{\circ} \mathrm{C}$, d pyridaben, e paraquat, $\mathbf{f} \mathrm{VC}, \mathbf{g ~ U V}$, and $\mathbf{h ~} \mathrm{HgCl}_{2}$. $\mathrm{CK}$ is the abbreviation for control check. The letters above the bars indicate significant differences $(P<0.01)$ according to the SAS software version 9.1. The data are the mean \pm SEM of three independent experiments. The letters above the columns indicate significant differences $(P<0.01)$ according to Duncan's multiple range test.

essential role in metabolism and development of most organisms (Zhou et al. 2015). These results revealed that AccTPI might be essential for the growth and development of bees. Additionally, the abdomen and thorax have higher AccTPI expression levels compared with other tissues (Figure 2b). Therefore, it is reasonable to deduce that AccTPI may be involved in differentiation or development relating to the mechanism of maturation, especially in the case of oxidation 

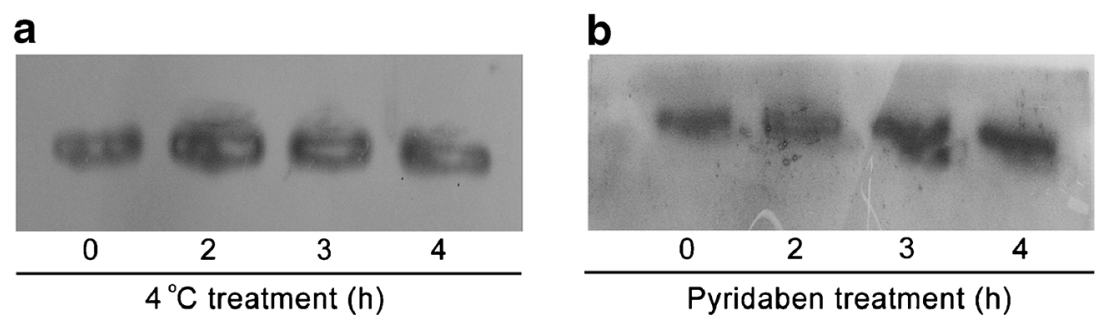

Pyridaben treatment $(h)$

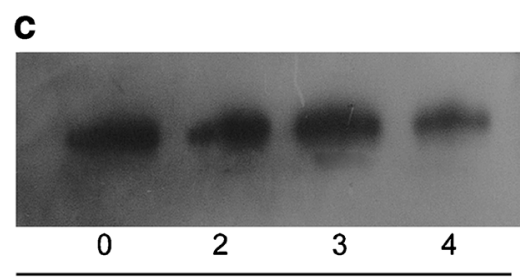

UV treatment $(h)$

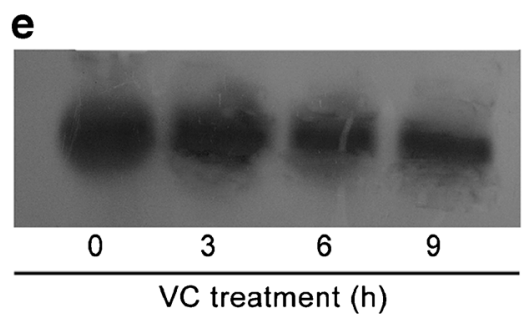

d

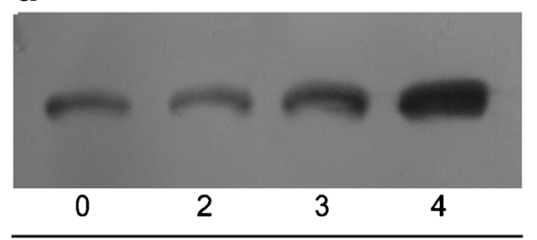

Methomyl treatment $(\mathrm{h})$

$\mathbf{f}$

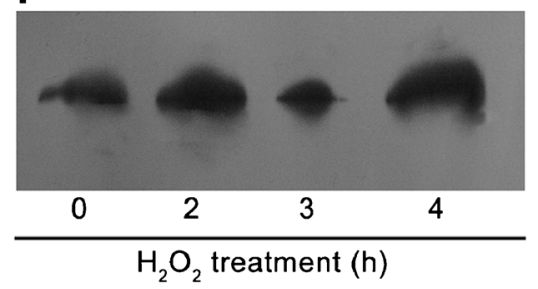

Figure 4. Western blot analysis of AccTPI. Western blot conditions include 15-day-old adult bees that were subjected to treatments with a $4{ }^{\circ} \mathrm{C}$, b pyridaben, c UV, d methomyl, e $\mathrm{VC}$ and $\mathbf{f} \mathrm{H}_{2} \mathrm{O}_{2}$. All lanes were loaded with an equivalent amount of extracted protein for each group.

resistance. Considering all of these results, we propose that AccTPI may be involved in organismal development and growth.

Previous studies have indicated that A. cerana cerana are stressed due to environmental changes and ROS (Yan et al. 2012). As the expression of AccTPI in A. cerana cerana due to exposure to oxidative stress has not yet been systematically reported, we investigated in this study the expression levels of AccTPI in response to various abiotic stresses. The results demonstrate that AccTPI may play an important role in protecting $A$. cerana cerana from oxidative stresses.

Temperature is an abiotic environmental factor that causes physiological changes in organisms and affects herbivorous insects (An and Choi 2010). In general, heat shock stress can result in polyamine oxidation to generate $\mathrm{H}_{2} \mathrm{O}_{2}$, and ROS act as key mediators of cold-induced apoptosis (Rauen et al. 1999). In the present study, the expression levels of AccTPI under 4 and $44{ }^{\circ} \mathrm{C}$ conditions were up-regulated following treatment. Western blot analysis of AccTPI under $4{ }^{\circ} \mathrm{C}$ (Figure $4 \mathrm{a}$ ) treatments confirmed the result, which demonstrates that AccTPI may play a vital role in response to extreme temperature, low temperature in particular. These results suggest that AccTPI may function by removing intracellular superoxide anions generated under high- or low-temperature conditions to protect honeybees from ROS damage. All of these results indicated that AccTPI may be associated with the prevention of the oxidative damage.

Oxidative stress can be induced by insecticides, temperature, and UV radiation (Kottuparambila et al. 2012). The role of pesticides in honeybee colony losses, with their sub-lethal and synergistic effects, has recently regained consideration (Boncristiani et al. 2012). TPI also plays an important role in response to adverse environmental 


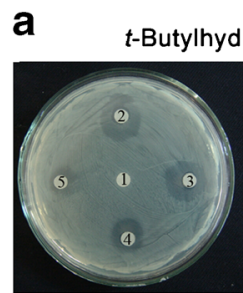

Control

b Cumene hydroperoxide

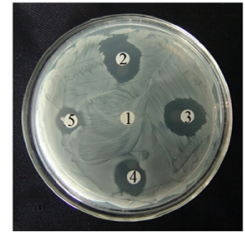

Control

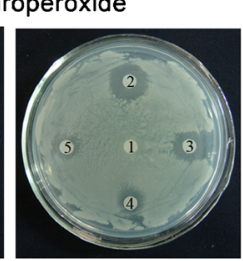

AccTPI
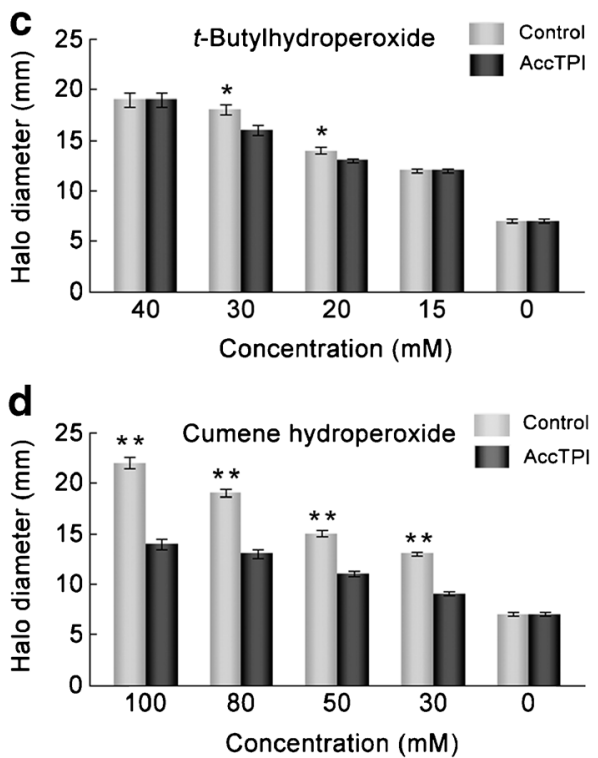

Figure 5. Disc diffusion assays for AccTPI. Filter discs soaked with different concentrations of a, c $t$ butylhydroperoxide and $\mathbf{b}, \mathbf{d}$ cumene hydroperoxide were placed on agar plates. The picture on the left corresponds with the numbers on the right. The data are the mean \pm SEM of three independent experiments. Statistical analysis was performed using Statistical Product and Service Solutions (SPSS) software version 19.0. Statistical significance was determined by unpaired Student's $t$ test; $* P<0.05, * * P<0.01$.

conditions (Hewitson et al. 2014). TPI is highly up-regulated under stress conditions in mammalian cells (Yamaji et al. 2004). In this study, paraquat and pyridaben treatments up-regulated TPI expression levels in A. cerana cerana workers. The expression of AccTPI was up-regulated under some pesticide treatments. All of these results demonstrate that AccTPI expression is induced by abiotic stress and that AccTPI may play a crucial role in survival under adverse environmental conditions.

There is much evidence that UV is one of the most ubiquitous environmental hazards for most animals which can cause oxidative stress and in turn lead to protein damage (Schauen et al. 2007). Many heavy metals can induce the formation of endogenous ROS (Liu et al. 2009a). $\mathrm{HgCl}_{2}$ treatment suppresses the expression of Acctpx-3 (Yao et al. 2013). When honeybees were treated with UV and $\mathrm{HgCl}_{2}$, expression levels of AccTPI were significantly down-regulated.
These results indicated that AccTPI may play various roles in dealing with various adverse conditions. The mRNA expression of AccTPI is specifically induced by certain stresses and repressed by others, and these results indicate that TPI is a mediator of the stressors. With an increase in the treatment concentration or an extension of the treatment time, the antioxidant system may temporarily be ineffective due to decreased expression. All of these results suggest that AccTPI is involved in resisting oxidative stress of $A$. cerana cerana restrictedly.

Western blot results for AccTPI under $\mathrm{H}_{2} \mathrm{O}_{2}$ and $\mathrm{VC}$ treatments verified that the expression is up-regulated and downregulated depending on the stressor. $\mathrm{H}_{2} \mathrm{O}_{2}$ induced ROS increase results in cell death caused by oxidative stress (Casini et al. 1986). $\mathrm{H}_{2} \mathrm{O}_{2}$-induced ROS increase results in cell death caused by oxidative stress (Goldshmit et al. 2001). Vitamin C, a well- 
known antioxidant, induces the decomposition of lipid hydroperoxides to endogenous genotoxins and results in DNA oxidative damage (Lee et al. 2001). The expression levels of AccTPI in VC treatments were significantly down-regulated. A possible explanation for this was that with the increase of stress, other stress-related genes might play important roles in cellular stress responses and the role of AccTPI might be weakened. The expression levels of the AccTPI gene in A. cerana cerana were varied in response to the oxidative stresses. Thus, AccTPI may have a significant influence on the response to environmental stresses.

To understand the role of honeybee TPI in defending against oxidative stresses, disc diffusion assays (Figure 5) were used to detect the level of protection of AccTPI in E. coli cells from oxidative stressors (cumene hydroperoxide and $t$-butylhydroperoxide). The model external pro-oxidants cumene hydroperoxide and $t$-butylhydroperoxide were used because they are more stable than $\mathrm{H}_{2} \mathrm{O}_{2}$ under the applied incubation conditions (Zhang et al. 2013). There were variations in the diameters of the death zones between the AccTPI-overexpressing bacteria and the control bacteria. Hence, we can conclude that AccTPI can significantly contribute to cellular resistance under oxidative stresses and that the AccTPI gene has antioxygenic properties. The purified recombinant AccTPI protein showed a capacity to protect DNA from oxidative damage. Taken together, our data provide very useful information for further understanding the mechanisms responding to environmental pressures in A. cerana cerana.

In conclusion, all of the results indicate that AccTPI may play an important role in the metabolism and antioxidant defense of honeybees. The unique functional characteristics, expression patterns, and potential physiological roles of AccTPI that were demonstrated in this study offer the basic knowledge for further studies. It is an eternal theme for the survival of living organisms to protection against oxidative stress.

\section{ACKNOWLEDGMENTS}

This work was financially supported by the earmarked fund for the China Agriculture Research System (No. CARS-45), the National Natural Science Foundation of China (No. 31172275), and the Shandong Province Agricultural Fine Varieties Breeding Projects (2014-2016).

Clonage et identification moléculaire du gène de la triose-phosphate isomérase extrait d'Apis cerana cerana et son rôle dans la réponse à des stress variés

stress abiotique / métabolisme / enzyme / développement / Apidae

Klonierung und molekulare Identifizierung des Triosephosphatisomerase-Gens von Apis cerana cerana und seine Rolle in der Antwort auf verschiedene

Stressfaktoren Triosephosphatisomerase / Apis cerana cerana / abiotischer Stress / Metabolismus

\section{REFERENCES}

Alaux, C., Ducloz, F., Crauser, D., Le Conte, Y. (2010) Diet effects on honeybee immunocompetence. Biol. Lett. 6, 562-565

An, M.I., Choi, C.Y. (2010) Activity of antioxidant enzymes and physiological responses in ark shell, Scapharca broughtonii, exposed to thermal and osmotic stress: effects on hemolymph and biochemical parameters. Comp. Biochem. Physiol. B Biochem. Mol. Biol. 155, 34-42

Boncristiani, H., Underwood, R., Schwarz, R., Evans, J.D., Pettis, J., van Engelsdorp, D. (2012) Direct effect of acaricides on pathogen loads and gene expression levels in honey bees Apis mellifera. J. Insect Physiol. 5, 613-620

Brennan, L.J., Keddie, B.A., Braig, H.R., Harris, H.L. (2008) The endosymbion Wolbachia pipientis induces the expression of host antioxidant proteins in a Aedes albopictus cell line. PLoS ONE 3, e2083

Burmeister, C., Luersen, K., Heinick, A., Hussein, A., Domagalski, M., Walter, R.D., Liebau, E. (2008) Oxidative stress in Caenorhabditis elegans: protective effects of the Omega class glutathione transferase (GSTO-1). FASEB J. 22, 343-354

Casini, A.F., Ferrali, M., Pompella, A., Maellaro, E., Comporti, M. (1986) Lipid peroxidation and cellular damage in extrahepatic tissues of bromobenzeneintoxicated mice. Am. J. Pathol. 123, 520-531

Chen, X., Yao, P., Chu, X., Hao, L., Guo, X., Xu, B. (2014) Isolation of arginine kinase from Apis cerana cerana 
and its possible involvement in response to adverse stress. Cell Stress Chaperones 20, 169-183

Chu, C., Lai, Y., Huang, H., Sun, Y. (2008) Kinetic and structural properties of triosephosphate isomerase from Helicobacter pylori. Proteins 71, 396-406

Elias-Neto, M., Soares, M.P., Simoes, Z.L., Hartfelder, K., Bitondi, M.M. (2010) Developmental characterization, function and regulation of a Laccase 2 encoding gene in the honey bee, Apis mellifera (Hymenoptera, Apinae). Insect Biochem. Mol. Biol. 40, 231-241

Felton, G.W., Summers, C.B. (1995) Antioxidant systems in insects. Arch. Insect Biochem. Physiol. 29, 187-197

Goldshmit, Y., Erlich, S., Pinkas-Kramarski, R. (2001) Neuregulin rescues PC12-ErbB4 cells from cell death induced by $\mathrm{H}_{2} \mathrm{O}_{2}$ regulation of reactive oxygen species levels by phosphatidylinositol 3-kinase. J. Biol. Chem. 49, 379-385

Helfert, S., Estevez, A.M., Bakker, B., Michels, P., Clayton, C. (2001) Roles of triosephosphate isomerase and aerobic metabolism in Trypanosoma brucei . Biochem. J. 357, 117-125

Hewitson, J.P., Ruckerl, D., Harcus, Y., Murray, J., Webb, L.M., Babayan, S.A., Allen, J.E., Kurniawan, A., Maizels, R.M. (2014) The secreted triosephosphate isomerase of Brugia malayi is required to sustain microfilaria production in vivo. PLoS Pathog. 10 (2), e1003030

Hrizo, S.L., Palladino, M.J. (2010) Hsp70- and Hsp90mediated proteasomal degradation underlies TPI ${ }^{\text {sugarkill }}$ pathogenesis in Drosophila . Neurobiol. Dis. 3, 676683

Imlay, J.A. (2003) Pathways of oxidative damage. Annu. Rev. Microbiol. 57, 395-418

Jia, H., Sun, R., Shi, W., Yan, Y., Li, H., Guo, X., Xu, B. (2014) Characterization of a mitochondrial manganese superoxide dismutase gene from Apis cerana cerana and its role in oxidative stress. J. Insect Physiol. 60, 68-79

Keller, L., Jemielity, S. (2006) Social insects as a model to study the molecular basis of ageing. Exp. Gerontol. 41, 553-556

Kottuparambila, S., Shinb, W., Brownc, M.T., Han, T. (2012) UV-B affects photosynthesis, ROS production and motility of the freshwater flagellate, Euglena agilis Carter. Aquat. Toxicol. 122-123, 206-213

Lee, S.H., Oe, T., Blair, I.A. (2001) Vitamin C-induced decomposition of lipid hydroperoxides to endogenous genotoxins. Science 292, 2083-2086

Li, H., Zhang, Y., Gao, Q., Cheng, J., Lou, B. (2008) Molecular identification of cDNA, immunolocalization, and expression of a putative odorant-binding protein from an Asian honey bee, Apis cerana cerana . J. Chem. Ecol. 34, 1593-1601

Liu, J., Qu, W., Kadiiska, M.B. (2009) Role of oxidative stress in cadmium toxicity and carcinogenesis. Toxicol. Appl. Pharmacol. 238, 209-214

Livak, K.J., Schmittgen, T.D. (2001) Analysis of relative gene expression data using real-time quantitative PCR and the 2 (-Delta C (T)) Method. Methods 25, 402408

Park, D., Jung, J.W., Choi, B.S., Jayakodi, M., Lee, J., Lim, J., Yu, Y., Choi, Y.S., Lee, M.L., Park, Y., Choi, I.Y., Yang, T.J., Edwards, O.R., Nah, G., Kwon, H.W. (2015) Uncovering the novel characteristics of Asian honey bee, Apis cerana, by whole genome sequencing. BMC Genomics 16, 1

Qin, Q., He, X., Tian, L., Zhang, S., Zeng, Z. (2012) Comparison of learning and memory of Apis cerana and Apis mellifera. J. Comp. Physiol. A. 198, 777786

Ralser, M., Heeren, G., Breitenbach, M., Lehrach, H., Krobitsch, S. (2006) Triosephosphate isomerase deficiency is caused by altered dimerization-not catalytic inactivity-of the mutant enzymes. PLoS One 1, e30

Rauen, U., Polzar, B., Stephan, H., Mannherz, H.G., de Groot, H. (1999) Cold-induced apoptosis in cultured hepatocytes and liver endothelial cells: mediation by reactive oxygen species. FASEB J. 13, 155-168

Roland, B.P., Stuchul, K.A., Larsen, S.B., Amrich, C.G., Vandemark, A.P., Celotto, A.M., Palladino, M.J. (2013) Evidence of a triosephosphate isomerase noncatalytic function crucial to behavior and longevity. J. Cell Sci. 126, 3151-3158

Schauen, M., Hornig-Do, H.T., Schomberg, S., Herrmann, G., Wiesner, R.J. (2007) Mitochondrial electron transport chain activity is not involved in ultraviolet A (UVA)-induced cell death. Free Radic. Biol. Med. 42, 499-509

Valko, M., Rhodes, C.J., Moncol, J., Izakovic, M., Mazur, M. (2006) Free radicals, metals and antioxidants in oxidative stress-induced cancer. Chem. Biol. Interact. $160,1-40$

Wang, J., Wei, D., Dou, W., Hu, F., Liu, W., Wang, J. (2013) Toxicities and synergistic effects of several insecticides against the oriental fruit fly (Diptera: Tephritidae). J. Econ. Entomol. 106, 970-978

Weinstock, G.M., Robinson, G.E., Gibbs, R.A., Worley, K.C., Evans, J.D., et al. (2006) Insights into social insects from the genome of the honeybee Apis mellifera. Nature 443, 931-949

Yamaji, R., Fujita, K., Nakanishi, I., Nagao, K., Naito, M. (2004) Hypoxic up-regulation of triosephosphate isomerase expression in mouse brain capillary endothelial cells. Arch. Biochem. Biophys. 423, 332-342

Yan, H., Meng, F., Jia, H., Guo, X., Xu, B. (2012) The identification and oxidative stress response of a zeta class glutathione S-transferase (GSTZ1) gene from Apis cerana cerana. J. Insect Physiol. 58, 782-791

Yao, P., Lu, W., Meng, F., Wang, X., Xu, B., Guo, X. (2013) Molecular cloning, expression and oxidative stress response of a mitochondrial thioredoxin peroxidase gene (AccTpx-3) from Apis cerana cerana . J. Insect Physiol. 59, 273-282

Zhang, Y., Yan, H., Lu, W., Li, Y., Guo, X., Xu, B. (2013) A novel Omega-class glutathione S-transferase gene in 
Apis cerana cerana: molecular characterisation of GSTO2 and its protective effects in oxidative stress. Cell Stress Chaperones 18, 503-516

Zhang, L., Yan, W., Wang, Z., Guo, Y., Yi, Y., Zhang, S., Zeng, Z. (2015) Differential protein expression analysis following olfactory learning in Apis cerana. J Comp Physiol A Neuroethol Sens Neural Behav Physiol 201, 1053-1061
Zhou, J., Liao, H., Li, S., Zhou, C., Huang, Y., Li, X., Liang, C., Yu, X. (2015) Molecular identification, immunolocalization, and characterization of Clonorchis sinensis triosephosphate isomerase. Parasitol. Res. 114, 3117-3124

Zinsser, V.L., Hoey, E.M., Trudgett, A., Tpison, D.J. (2013) Biochemical charaterisation of triosephosphate isomerase from the liver fluke Fasciola hepatica. Biochimie 95, 2182-2189 